\title{
In Response to the Letter to the Editor regarding "Pulsed radiofrequency in pain management"
}

Thank you for taking interest in reading our article and for providing such valuable input. Yes, according to our study, we found that pulsed radiofrequency (PRF) group, compared to the intraarticular depot methylprednisolone injection group, has comparably prolonged pain relief outcome after 3 and 6 months.

We also want to mention that, to our knowledge, our study is the first of its type to compare PRF of articular branches of the sacroiliac joint and L5/S1 in the management of sacroiliac joint pain. The study by Roy et al (1) evaluated the efficacy of combined treatment with medial branch radiofrequency neurotomy and steroid block in lumbar facet joint arthropathy in which steroid was injected after radiofrequency ablation of medial branch neurotomy. In this study, conventional radiofrequency was used $\left(85^{\circ} \mathrm{C}\right.$ for 90 seconds) and steroid was injected after the radiofrequency lesioning, probably to prevent neuritis. However, in our study, we have used PRF that works on a different mechanism in which electrical current reversibly disrupts impulse transmission in small unmyelinated $C$ pain fibers without neuroablation or thermal destruction (2). Therefore, we didn't inject steroid post-PRF ablation.

We really appreciate and accept the suggestion to continue the follow-up for 1 year or more, and we could also include an arm of PRF + steroid and compare all 3 to have results that would help in future evidence for clinical practice.

\author{
Samarjit Dey, MD \\ Associate Professor \\ Department of Anaesthesiology \\ AlIMS Raipur CG, India \\ E-mail: drsamar0002@gmail.com \\ Kaustuv Dutta, MD \\ Consultant, Neuroanaesthesia \\ Apollo Hospitals \\ Bangalore, India \\ E-mail: kaustuv.dutta@gmail.com
}

\section{REFERENCES}

1. Roy C, Chatterjee N, Ganguly S, Sengupta R. Efficacy of combined treatment with medial branch radiofrequency neu- rotomy and steroid block in lumbar facet joint arthropathy. J Vasc Interv Radiol 2012; 23:1659-1664.
2. Das G, Dey S. Pulsed radio frequency in pain management. Indian J Pain 2013; 27:111-113 